Pacific Journal of Mathematic 


\title{
MANIFOLDS ADMITTING TAUT HYPERSPHERES
}

\author{
JAMES J. HEBDA
}

\begin{abstract}
The definition of taut submanifolds in Euclidean space is extended to submanifolds of an arbitrary complete Riemannian manifold. Manifolds containing a tautly embedded hypersphere are characterized up to homeomorphism. Also, a partial result in this direction is proved for manifolds containing a tautly embedded sphere of arbitrary codimension.
\end{abstract}

1. Taut submanifolds have received much attention in recent years [1], [3], [6], [7]. There the emphasis is on characterizing the taut submanifolds of a particular ambient space, usually Euclidean space, although there are studies involving hyperbolic space and complex projective space as well [4], [5]. In this paper the subject is approached from a different perspective: to characterize the ambient space given that it contains certain taut submanifolds. For example:

THEOREM 1. A complete simply connected Riemannian manifold of dimension $n$ that admits a taut embedding of $S^{n-1}$ is either homeomorphic to $\boldsymbol{S}^{n}$, diffeomorphic to $\boldsymbol{R}^{n}$, or diffeomorphic to $\boldsymbol{S}^{n-1} \times \boldsymbol{R}$.

In a Euclidean sphere or in a complete, simply-connected Riemannian manifold without conjugate points, every geodesic sphere is taut. The converse is also true.

THEOREM 2. Suppose a complete Riemannian manifold has the property that about every point some small geodesic sphere is taut. Then the manifold is either simply connected without conjugate points or isometric to a Euclidean sphere.

2. Let $M$ be a complete Riemannian manifold and $N \subset M$ a proper submanifold. In particular, $N$ is a closed submanifold with the subspace topology. For each $p \in M$, we define the function $L_{p}: N \rightarrow \boldsymbol{R}$ by $L_{p}(x)=[d(x, p)]^{2}$ where $x \in N$ and $d$ is the distance function on $M$. We say $N$ is taut if for almost every $p \in M$ and almost every $r>0$ the homeomorphism

$$
i_{*}: H_{*}\left(L_{p}^{-1}([0, r])\right) \longrightarrow H_{*}(N)
$$

induced by inclusion is injective, where the homology coefficients are in some field. Because of Lemma 2.8 on page 705 of [3], this definition coincides with the definition for taut submanifolds of 
Euclidean space. It roughly states that on a dense set of $p \in M$, the function $L_{p}$ has the least possible number of critical points.

$L_{p}$ is a continuous function which is smooth at the points of $N$ that are not in the cut locus $C(p)$ of $p$. At these points, one has a characterization of a critical point of $L_{p}$ and its index in terms of the focal points of $N$ [12].

Proposition 1. A point $x_{0} \in N, x_{0} \notin C(p)$ is a critical point of $L_{p}$ if and only if the unique minimizing geodesic from $p$ to $x_{0}$ is perpendicular to $N$ at $x_{0}$. Furthermore, its index is the number of focal points of $N$ between $x_{0}$ and $p$, counted with multiplicity, along this geodesic, and its nullity is the multiplicity of $p$ as a focal point.

Proof. The first statement follows from the first variation formula, and the second is essentially the finite dimensional approximation of the path space by broken geodesics, e.g., [10], page 160, where broken geodesics are unnecessary since we evade the cut locus of $p$.

The nature of the critical points of $L_{p}$ and $L_{p^{\prime}}$ are similar if $p$ and $p^{\prime}$ are close together.

Proposition 2. (i ) Let $x_{0} \in N, x_{0} \notin C(p)$ and $U$ be a neighborhood of $x_{0}$ in $N$. If $x_{0}$ is a nondegenerate critical point of $L_{p}$ with index $\lambda$, then for all $p^{\prime}$ sufficiently near $p, L_{p^{\prime}}$ has a nondegenerate critical point in $U$ of index $\lambda$.

(ii) Let $K \subset N$ be a compact subset and $U$ a relatively compact neighborhood of $K$ in $N$. If $L_{p}$ has a relative minimum on $K$, i.e., $L_{p}$ is constant on $K$ and $L_{p}(x)>L_{p}(K)$ for all $x \in U-K$, then for all $p^{\prime}$ sufficiently near $p, L_{p^{\prime}}$ attains a relative minimum in $U$. A similar statement is true for relative maximums.

Proof. (i) is a consequence of the fact that nearby geodesics have the same index. The proof of (ii) is analogous to Lemma 5.1 of [11].

In what follows, we will be concerned with taut embeddings of spheres $S^{k}$. In this case, the tautness condition and Proposition 2 imply that a relative minimum of $L_{p}$ is an absolute minimum, that a relative maximum is an absolute maximum, and that the index of any nondegenerate critical point must be either 0 or $k$.

3. In this section we prove Theorem 1 . We begin with the 
following proposition.

Proposition 3. Let $M$ be a complete Riemannian manifold and $N$ a taut connected hypersurface which separates $M$. Let $\gamma$ be a geodesic starting at $x_{0} \in N$ perpendicularly to $N$. If there exists a cut point of $x_{0}$ along $\gamma$, then the first focal point of $N$ along $\gamma$ occurs between $x_{0}$ and the eut point.

Proof. Since $N$ separates $M, M-N$ consists of two connected components. Furthermore, being taut, the relative minimums of $L_{p}$ are absolute minimums. Let $p_{0}$ be the cut point of $x_{0}$ along $\gamma$ and suppose the first focal point of $N$ along $\gamma$ does not occur between $x_{0}$ and $p_{0}$. Then, by Proposition 1 , for any $p$ along $\gamma$ between $x_{0}$ and $p_{0}, L_{p}$ has a nondegenerate relative minimum at $x_{0}$, there being no focal points between $x_{0}$ and $p$. This is an absolute minimum. Thus for all $x \in N, d(x, p) \geqq d\left(x_{0}, p\right)$ and by taking limits as $p$ approaches $p_{0}, d\left(x, p_{0}\right) \geqq d\left(x_{0}, p_{0}\right)$ for all $x \in N$. This shows that the segment of $\gamma$ between $x_{0}$ and $p_{0}$ is contained in one of the components of $M-N$. (If it wasn't we could find a point of $N$ closer to $p_{0}$ than $x_{0}$.) Extend $\gamma$ on the "other side" of $N$ and take a point $q$ on $\gamma$ close enough to $N$ so that there is no focal point between $x_{0}$ and $q$, and $q$ lies on the opposite side of $N$ from $p_{0}$. As before, $L_{q}$ attains its absolute minimum at $x_{0}$. Thus $d(x, q) \geqq d\left(x_{0}, q\right)$ for all $x \in N$. Now, the segment of $\gamma$ between $q$ and $p_{0}$ is not minimizing, since it contains the cut point $x_{0}$ of $p_{0}$. Hence, by completeness of $M$, there is a minimizing segment $\sigma$ connecting $q$ to $p_{0}$. Since $N$ separates $M, \sigma$ intersects $N$ at some point $x$. Hence $d\left(p_{0}, q\right)=$ $d\left(p_{0}, x\right)+d(x, q) \geqq d\left(p_{0}, x_{0}\right)+d\left(x_{0}, q\right)=$ length of the segment of $\gamma$, which contradicts the nonminimality of $\gamma$. Therefore we have shown the first focal point of $N$ occurs between $x_{0}$ and $p_{0}$.

REMARK. By [13], if $M$ is simply connected, every properly embedded, connected hypersurface of $M$ separates $M$. Thus the hypothesis that $N$ separates $M$ can be dropped from Proposition 3 if $M$ is simply connected.

In view of this remark, Theorem 1 is a consequence of the following Theorem $1^{\prime}$.

THEOREM 1'. Let $M$ be a complete Riemannian manifold of dimension $n$ that admits a taut embedding of $S^{n-1}$ which separates $M$, then $M$ is either homeomorphic to $S^{n}$, diffeomorphic to $\boldsymbol{R}^{n}$, or diffeomorphic to $S^{n-1} \times \boldsymbol{R}$.

Proof. Let $N$ be the taut embedding of $S^{n-1}$ separating $M$. 
Thus by Proposition 3, we can conclude that the first focal points occur prior to the cut points. The results of $\S 2$ allow us to conclude that a first focal point must be of multiplicity $n-1$. (If not, by choosing a point $p$ slightly beyond the first focal point on the geodesic, the function $L_{p}$ would have a nondegenerate critical point of index $\lambda$ with $0<\lambda<n-1$.) If $p_{0}$ is the first focal point of $N$ along $\gamma$ starting perpendicularly at $x_{0} \in N$, then $L_{p_{0}}$ is constant on $N$. For taking $p$ between $x_{0}$ and $p_{0}, L_{p}$ has a minimum at $x_{0}$, and $p$ with $p_{0}$ between $x_{0}$ and $p, L_{p}$ has a maximum at $x_{0}$. Since these are absolute extrema, on taking limits as $p$ approaches $p_{0}$ we find $L_{p_{0}}$ is constant. Since the multiplicity of the first focal points is constant, the first focal locus is an $n-1$ dimensional submanifold of the normal bundle of $N$ [9]. (A generalization of Warner's regular conjugate locus [14].) Furthermore, we have essentially shown that the distance to the first focal point is constant on each side of $N$. (We are in a situation analogous to the regular spherical conjugate loci of [15].) Hence, the components of the first focal locus in the normal bundle get identified each to a single point, since the focal points are of maximal multiplicity. Now the first focal locus has at most one component on each side of $N$. Hence this leaves three possibilities, either there are two focal points of $N$, one on either side; there is only one focal point; or there are no focal points. Now, two geodesics starting perpendicularly to $N$ cannot meet until at least one of them has reached the first focal point, otherwise if $p$ is the point of intersection, $L_{p}$ has two distinct minima in contradiction to the tautness of $N$. This says that the exponential map of the normal bundle of $N$ into $M$ is one-one on the set of normal vectors shorter than the distance to the focal point. Thus if there are no focal points the exponential map gives a diffeomorphism of the normal bundle of $N$ to $M$, hence $M$ is diffeomorphic to $S^{n-1} \times \boldsymbol{R}$. If there is only one focal point, $p_{0}$, then $N$ is a geodesic sphere about $p_{0}$ and since the focal points of a geodesic sphere about a point correspond to the conjugate points of that point, $p_{0}$ has no conjugate points. Furthermore, since the exponential map on the normal bundle of $N$ is one-one up to the focal points, the exponential map at $p_{0}$ is one-one. Hence, $M$ is diffeomorphic to $T_{p_{0}} M \cong \boldsymbol{R}^{n}$ under the exponential map at $p_{0}$. Lastly, if there are two focal points, let $p_{0}$ be one of them. Then, $N$ is a geodesic sphere about $p_{0}$, and, by the same reasoning as in the previous case, the first conjugate points to $p_{0}$ occur at constant distance and no pair of geodesics emitted from $p_{0}$ meet before this conjugate point which is of multiplicity $n-1$. We can conclude that $M$ is a Blaschke manifold at $p_{0}$, and is homeomorphic to $S^{n}$. (See Theorem 5.43 of [2] and Theorem 3.1 of [15].) 
4. In order to prove Theorem 2, suppose that for every $p \in M$, some geodesic sphere $S(p, r)$ about $p$ of radius $r$ less than the injectivity radius at $p$ is taut. Certainly, every such geodesic sphere separates $M$, and $p$ is a focal point of $S(p, r)$. This leaves two possibilities. Either $M$ is diffeomorphic to $\boldsymbol{R}^{n}$ and every $p \in M$ has no conjugate points, or $M$ is homeomorphic to $S^{n}$ and is a Blaschke manifold at every point. In the second case, Berger's theorem in Appendix D of [2], states that $M$ is isometric to a Euclidean sphere. This proves Theorem 2 .

5. In this section we sketch a partial result for manifolds admitting taut spheres of arbitrary codimension. The following was proved in [8] with an extra assumption of simple connectivity.

THEOREM 3. Let $M$ be a compact Riemannian manifold admitting a taut embedding of $S^{k}$. Suppose that the first focal point on every geodesic starting at $x_{0} \in S^{k}$ perpendicularly to $S^{k}$ occurs between $x_{0}$ and its cut point. Then $M$ is homeomorphic to a sphere.

Proof. As in $\S 3$, we show that the multiplicity of a first focal point is $k$, that for every first focal point $p_{0}, L_{p_{0}}$ is constant on $S^{k}$, and that a pair of geodesics starting perpendicularly to $S^{k}$ do not meet until at least one of them has reached the first focal point. If we consider the first focal locus $X$ of $S^{k}$ in the normal bundle, it can be shown that $X$ is a hypersurface of the normal bundle and that the exponential map restricted to $X$ is a submersion into $M$ [9]. Since $M$ is compact, every geodesic has cut points, and we may conclude that $X$ is compact. Hence, using the submersion, $\exp (X)=K$ is a compact submanifold of $M$ of dimension $n-k-1$. Futhermore, one can prove that $M$ is expressible as the union of the normal disk bundle of $S^{k}$ and the normal disk bundle of $K$ pasted together by a diffeomorphism of their boundaries. These common boundaries may be identified with $X$ in such a way that the exponential map restricted to $X$ is the projection of the normal sphere bundle onto $K$. The map $\left(\pi\right.$, exp): $X \rightarrow S^{k} \times K$, where $\pi$ is the restriction to $X$ of the projection of the normal bundle of $S^{k}$ onto $S^{k}$, is a diffeomorphism because, first of all, it is clearly an immersion of manifolds of the same dimension and compactness implies it is onto, and secondly, if it was not one to one, one could find two minimizing geodesics from a point $x_{0} \in S^{k}$ to a focal point $p_{0} \in K$, contradicting the condition that the first focal point occurs before the cut point. Similarly, for any $x_{0} \in S^{k}$, exp: $\pi^{-1}\left(x_{0}\right) \rightarrow K$ is a diffeomorphism. Therefore, $K$ is diffeomorphic to $S^{n-k-1}$ since $\pi^{-1}\left(x_{0}\right)$ is diffeomorphic to $S^{n-k-1}$, and $X$ is diffeomorphic to $S^{k} \times S^{n-k-1}$. By 
the mapping cylinder construction, we see that the normal disk bundle of $S^{k}$ is homeomorphic to $S^{k} \times D^{n-k}$, that the normal disk bundle of $K$ is homeomorphic to $D^{k+1} \times S^{n-k-1}$, and that the pasting map on the boundaries corresponds to the identity map. However, $S^{k} \times D^{n-k}$ and $D^{k+1} \times S^{n-k-1}$ pasted together via the identity map on the boundaries is the sphere $S^{n}$.

REMARK. The condition on the focal points is not generally satisfied by taut spheres when the codimension is greater than one. A easy example is the following. Let $M=S^{m} \times S^{n}$. Let $S^{k}$ be a great sphere in $S^{m}$ with $k<m$, and $p \in S^{n}$. The submanifold $N=$ $S^{k} \times\{p\}$ is taut. However, along the geodesic $\gamma=\left(x_{0}, \gamma_{0}\right)$, where $\gamma_{0}$ is a geodesic in $S^{n}$ starting at $p$ and $x_{0} \in S^{k}$, the first focal point of $N$ and the cut point to $\left(x_{0}, p\right)$ coincide.

\section{REFERENCES}

1. T.F. Banchoff, The spherical two-piece property and tight surfaces in spheres, J. Differential Geometry, 4 (1970), 193-205.

2. A. L. Besse, Manifolds All of Whose Geodesics are Closed, Springer-Verlag, BerlinHeidelberg-New York, 1978.

3. S. Carter and A. West, Tight and taut immersions, Proc. London Math. Soc., 25 (1972), 701-720.

4. T. Cecil, A characterization of metric spheres in hyperbolic space by Morse theory, Tôhoku Math. J., 26 (1974), 341-351.

5. - Geometric applications of critical point theory submanifolds of complex projective space, Nagoya Math. J., 55 (1974), 5-31.

6. - Taut immersions of noncompact surfaces into a Euclidean 3-space, J. Differential Geometry, 11 (1976), 451-459.

7. T. Cecil and P. Ryan, Focal sets, taut embeddings and the cyclides of Dupin, Math. Ann., 236 (1978), 117-190.

8. J.J. Hebda, Submanifolds, focal points and Morse functions, Doctoral Thesis, University of Chicago, 1979.

9. The regular focal locus, to appear.

10. N.J. Hicks, Notes on Differential Geometry, Van Nostrand Reinhold Company, London, 1971.

11. N. H. Kuiper, On surfaces in euclidean three-space, Bull. Soc. Math. de Belgique, 12 (1960), 5-22.

12. J. Milnor, Morse Theory, Princeton University Press, Princeton, 1963.

13. H. Samelson, Orientability of Hypersurfaces in $\boldsymbol{R}^{n}$, Proc. Amer. Math. Soc., 22 (1969), 301-302.

14. F. W. Warner, The conjugate locus of a Riemannian manifold, Amer. J. Math., 87 (1965), 575-604.

15. Conjugate loci of constant order, Ann. of Math., 86 (1967), 192-212.

Received October 7, 1980 and in revised form March 10, 1981.

Michigan State University

EAST LANSING, MI 48824 


\section{PACIFIC JOURNAL OF MATHEMATICS}

\section{EDITORS}

DONALD BABBITT (Managing Editor)

University of California

Los Angeles, California 90024

Hugo RossI

University of Utah

Salt Lake City, UT 84112

C. C. MOORE and ANDREW OGG

University of California

Berkeley, CA 94720

\section{J. DugundJI}

Department of Mathematics University of Southern California Los Angeles, California 90007

R. Finn and J. Milgram Stanford University

Stanford, California 94305

\section{ASSOCIATE EDITORS}
R. ARENS
E. F. BeCKENBACH
B. H. Neumann
F. WoLF
K. YoSHIDA

\section{SUPPORTING INSTITUTIONS}

UNIVERSITY OF ARIZONA

UNIVERSITY OF BRITISH COLUMBIA

CALIFORNIA INSTITUTE OF TECHNOLOGY

UNIVERSITY OF CALIFORNIA

MONTANA STATE UNIVERSITY

UNIVERSITY OF NEVADA, RENO

NEW MEXICO STATE UNIVERSITY

OREGON STATE UNIVERSITY
UNIVERSITY OF OREGON

UNIVERSITY OF SOUTHERN CALIFORNIA

STANFORD UNIVERSITY

UNIVERSITY OF HAWAII

UNIVERSITY OF TOKYO

UNIVERSITY OF UTAH

WASHINGTON STATE UNIVERSITY

UNIVERSITY OF WASHINGTON 


\section{Pacific Journal of Mathematics}

\section{Vol. 97, No. $1 \quad$ January, 1981}

Charles A. Asmuth and Joe Repka, Tensor products for $S L_{2}(\mathfrak{k})$. II.

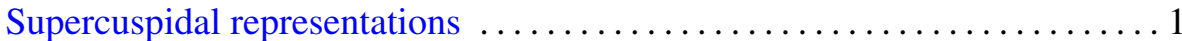

Joseph Barback, On finite sums of regressive isols . ................. 19

Matthew G. Brin and Daniel Russell McMillan, Jr., Generalized

three-manifolds with zero-dimensional nonmanifold set ............29

Kun Soo Chang, Converse measurability theorems for Yeh-Wiener space . . . 59

Christopher Brian Croke, A "maximal torus" type theorem for complete

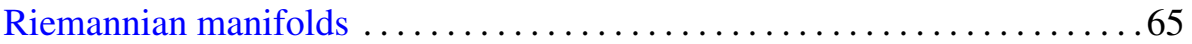

Gustave Adam Efroymson, Sums of squares in planar Nash rings . . . . . . 75

John Robert Fisher, Axiomatic radical and semisimple classes of rings . . . .81

Betty Kvarda, Consecutive integers for which $n^{2}+1$ is composite .......93

Roosevelt Gentry, New diagram proofs of the Hausdorff-Young theorem

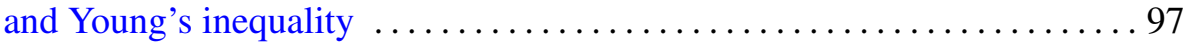

Patrick M. Gilmer, Topological proof of the $G$-signature theorem for $G$

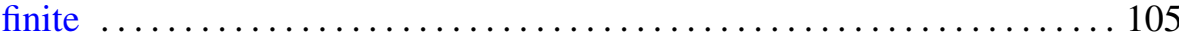

Chung Wei Ha, A noncompact minimax theorem .................. 115

James J. Hebda, Manifolds admitting taut hyperspheres ................ 119

Takayuki Kawada, Sample functions of Pólya processes ............. 125

Peter K. F. Kuhfittig, Common fixed points of nonexpansive mappings by

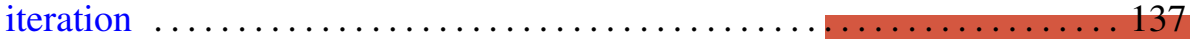

James Thomas Loats and Judith Roitman, Almost rigid Hopfian and dual

Hopfian atomic Boolean algebras .......................... 141

Roger McCann, On embedding semiflows into a radial flow on $l_{2} \ldots \ldots \ldots 151$

John McDonald, Closed orbits of convex sets of operators on the disk

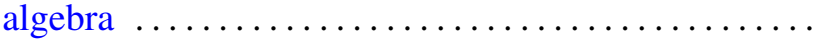

Mark D. Meyerson, Convexity and the table theorem .............. 167

Arnold William Miller, Generic Souslin sets . . . . . . . . . . . . . . . . 171

Takemi Mizokami, On the closed images of paracomplexes $\ldots \ldots \ldots \ldots \ldots 3$

Jagannadham Venkata Pakala and Thomas Stephen Shores, On

compactly packed rings $\ldots \ldots \ldots \ldots \ldots \ldots \ldots \ldots \ldots$

Andrew Pletch, Strong completeness in profinite groups

Wilbur Carrington Whitten, Inverting double knots

James Juei-Chin Yeh, Existence of strong solutions for stochastic

differential equations in the plane 